\title{
Is an increase in probability always an increase in evidential support?
}

\author{
Artūrs Logins, University of Zurich \\ [This is a post-peer-review, pre-copyedit version of an article published in Erkenntnis. The final authenticated version \\ is available online at: http://dx.doi.org/10.1007/s10670-020-00241-4]
}

\begin{abstract}
Peter Achinstein has argued at length and on many occasions that the view according to which evidential support is defined in terms of probability-raising faces serious counterexamples and, hence, should be abandoned. Proponents of the positive probabilistic relevance view have remained unconvinced. The debate seems to be in a deadlock. This paper is an attempt to move the debate forward and revisit some of the central claims within this debate. My conclusion here will be that while Achinstein may be right that his counterexamples undermine probabilistic relevance views of what it is for $e$ to be evidence that $h$, there is still room for a defence of a related probabilistic view about an increase in being supported, according to which, if $\mathrm{p}(h \mid e)>\mathrm{p}(h)$, then $h$ is more supported given $e$ than it is without $e$. My argument relies crucially on an insight from recent work on the linguistics of gradable adjectives.
\end{abstract}

\section{Introduction}

According to one popular approach within philosophy of confirmation, evidential support has to be defined in probabilistic terms. Its detractors, however, remain unconvinced. The debate has reached a stalemate. The aim of the present article is to move the debate forward. The main task that I will undertake here is to propose a novel examination of one particularly trenchant line of objection to the probabilistic approach, a famous argument proposed by Peter Achinstein (1978, 1983, 2000, 2001, 2004, 2013).

The argument in question is based on our common-sense intuitions about some cases where, allegedly, a proposition provides an increase in probability for a given hypothesis, and yet the same proposition is not evidence that the hypothesis in question is true. Many have tried to explain away the intuitions that Achinstein has brought to light (such as, for instance, by an appeal to conversational implicatures, cf. Maher 1996). I think that such a dismissal of the relevant intuitions is misguided. If the notion of evidence and evidential support in science has anything in common with its ordinary understanding, then Achinstein's argument cannot be dismissed from the outset.

At the end of the day, however, I think we should conclude that both parties are partially right 
and partially wrong. If we focus on the positive form of 'being evidentially supported' or 'being evidence that $h$ ' in its ordinary sense, then Achinstein seems to be right in claiming that $e$ being evidence that $h$ cannot be fully captured by mere appeal to an increase in probability. Probabilist views that claim otherwise are problematic. However, if we focus on comparative forms, e.g. ' $h$ is more supported given $e$, than without $e$, then there is still a room for probabilists to escape Achinstein's argument, or so I will argue. More specifically, I will suggest that Achinstein hasn't established that comparative evidential support cannot be understood in terms of probabilistic relevance, since a central premise in what appears to be Achinstein's main argument towards that more specific conclusion is problematic. The premise in question relies on a mistaken assumption.

I will not question here what appears to be Achinstein's central claim, namely, that $e$ being evidence that $h$ cannot be fully understood in terms of an increase in probability. This article aims to show that the view that an increase in probabilities entails an increase in comparative support escapes Achinstein's counterexamples. In a sense, then, my proposal amounts to a new conciliatory suggestion. The central intuition from Achinstein's approach might be right. Our common-sense judgements about cases of increase in probability not always amounting to something being evidence that the hypothesis is true might be correct. However, this is not to say that the probabilistic accounts are not interesting and useful tools for thinking about science and some aspects of confirmation and comparative support in particular. What our discussion will show, I hope, is that as long as evidential support in science has anything to do with the ordinary, common-sense understanding of evidential support, being evidentially supported cannot be merely reduced to an increase in probability, even though Achinstein's arguments don't establish that comparative evidential support cannot be understood in terms of probabilistic relevance.

In what follows, I first introduce Achinstein's cases and present his overall argumentative strategy. I then present a line of reply from a probabilist perspective (alongside some further remarks and specifications of the probabilist view). Third, I introduce (a possible reconstruction of) a more specific argument that might be implicit in Achinstein's texts, against my new line of reply to his counterexamples. Once the structure of this implicit argument has been made explicit I will show where exactly it seems to go wrong. In making my case I will appeal to some recent work on the linguistics of gradable adjectives.

\section{The focus of Achinstein's criticism}

Peter Achinstein has argued in length and on many occasions against probabilistic relevance accounts of evidential support. More specifically, he has objected to the view according to which $e$ is evidence 
that $h$ if and only if $\mathrm{p}(h \mid e)>\mathrm{p}(h)$. In other words, Achinstein has objected to the view that individually necessary and conjointly sufficient conditions for $e$ to be evidence that $h$ are that the probability of $h$ given $e$ is higher than the probability of $h$ without $e$. Achinstein thinks that an increase in probability is neither sufficient nor necessary for $e$ being evidence that $h .{ }^{1}$ Consider these passages from Achinstein, where he specifies his target and sets out his general argumentative strategy:

According to one probability definition e is potential evidence that $\mathrm{h}$ if and only if the probability of $\mathrm{h}$ given e is greater than the prior probability of $\mathrm{h}$ :

(1a) $e$ is potential evidence that $h$ if and only if $p(h, e)>p(h)$.

Or, if $\mathrm{b}$ is background information,

(1b) $e$ is potential evidence that $h$ if and only if $p(h, e \& b)>p(h, b)$.

A definition of this sort is offered by many writers. [...] However, despite its widespread acceptance it cannot possibly be correct if 'evidence' and 'probability' are being used as they are in ordinary language or science. [...] I shall [...] note three types of counterexamples. The first shows that an increase in probability is not sufficient for evidence, the second and third that it is not necessary. (Achinstein 1983: 150-151). ${ }^{23}$

And:

Carnap offers two different probabilistic definitions of the former [i.e. "qualitative" evidence]. The first, by far the most popular with probabilists, states that $e$ is evidence that $h$ if and only if $e$ increases h's probability:

[...] First Probability Definition of Evidence (increase in probability): $e$ is evidence that $h$ if and only if $p(h / e)>p(h)$,

${ }^{1}$ Achinstein also thinks that while a high probability of $h$ given $e$ is necessary, it is not sufficient for $e$ being evidence that $h$; see, for instance, Achinstein (2001: 71). The focus of the present discussion is on the probabilistic relevance views of evidential support, not on the high probability view of $e$ being evidence that $h$, according to which $e$ is evidence that $h$ if and only if the probability of $h$ given $e$ is higher than some specific threshold of high probability, e.g. .5, .75 or .9.

2 The subtleties of Achinstein's view on 'potential' evidence are, I suggest, not central for our purposes here. Similarly, for what matters here, we can focus on the simple relevance view without always making explicit the reference to possible background information.

${ }^{3}$ Note that in later writings (starting from 1983) Achinstein's formulation of the view in (1b) includes explicitly a reference to background information. See, for instance: "Probabilists may then relativize evidence statements to some set $b$ of background assumptions and say that (7) $e$ is evidence that $h$, given $b$, if and only if $p(h / e \& b)>p(h / b)$." (Achinstein 2001: 46). Thanks to an anonymous reviewer for drawing my attention to this aspect. 
where $p(h / e)$ means the probability of $h$, given $e$ (or on the assumption of $e$ ). This is called the "positive relevance" definition, where $e$ is relevant to $h$ if $e$ changes $h$ 's probability, and it is positively relevant if it increases it. (Achinstein 2001: 45).

It is important to stress that, as we see from these quotations, Achinstein's main focus is on what it is for $e$ to be evidence that $h$. That is, he is both formulating his probabilistic opponents as specifying conditions about $e$ being evidence that $h$ in probabilistic terms, and appealing to commonsense considerations about $e$ being evidence that $h$ in his attacks on these probabilistic views. Achinstein presents the main debate with a focus on the positive form of 'evidence that' rather than comparative constructions or constructions that have degree modifiers. For instance, Achinstein is not focusing on what is expressed by 'more evidence/support' nor 'some evidence' constructions. ${ }^{4}$ The main focus of Achinstein's criticism is the view that defines what it is for $e$ to be evidence that $h$ in terms of an increase in probability (i.e. $\mathrm{p}(h \mid e)>\mathrm{p}(h)$ ). Furthermore, Achinstein also avoids the 'evidence for' constructions in formulating the views of his opponents. He is not presenting his target as a view that specifies that $e$ is evidence for $h$ if and only if some probabilistic constraints are satisfied. His main focus is on claims that ' $e$ is evidence that $h$ iff...'. However, at least some proponents of probabilistic views who present their approach in terms of 'evidence for' constructions take it that they and Achinstein are discussing the same thing, and hence assume, roughly, the equivalence of 'evidence for' and 'evidence that' constructions. ${ }^{5}$ Finally, it is worth noticing that in order for Achinstein's argument to apply against some of the most prominent contemporary versions of Bayesian accounts, we also have to assume that when Achinstein talks about something being

${ }^{4}$ More precisely, a central focus of Achinstein's work over the years has been on what it is for $e$ to be evidence that $p$. However, he has also written on topics related to comparative aspects of evidence. In a paper on stronger evidence (Achinstein 1994), he does consider the question of what makes a piece of evidence $e 1$ stronger evidence (for $h$ ) than another piece of evidence $e 2$ (for $h$ ). Achinstein's main thesis there is that a probabilistic account (like Carnap's) of stronger evidence cannot be maintained. The focus is on the view according to which "Where $e_{l}$ and $e_{2}$ are both evidence for $h$, given $b, e_{l}$ is stronger evidence than is $e_{2}$ iff $p\left(h / e_{l} \& b\right)>p\left(h / e_{2} \& b\right)$ " (Achinstein 1994: 331). However, the discussion there is still closely related to Achinstein's objections to probabilistic views of evidence that $h$ (for instance, in his appeal to evidence being a reason to believe, see: "These combined results provide a reason for believing $h$ that is better, more convincing, than that provided by the first study alone" (Achinstein 1994: 335). We will come back to Achinstein's appeal to good reasons and reasonable belief in characterizing evidence in what follows.

${ }^{5}$ See, for instance, Patrick Maher, a prominent critic of Achinstein's proposal: "As we see from this example, Achinstein talks of evidence that a hypothesis is true, whereas I talk of evidence for a hypothesis. For Achinstein's objections to be relevant to the account of confirmation defended here, this difference needs to be regarded as merely stylistic and I will so regard it" (Maher 1996, footnote 11). 
evidence that $h$ and when Bayesians talk about $e$ confirming/supporting $h$ they are talking about the same thing. 6

Now that we have taken the precaution of specifying the main target of Achinstein's counterexamples, we are in a position to introduce the examples themselves. Here is one of the most famous of Achinstein's examples against the claim that an increase in probability is sufficient for $e$ to be evidence that $h:^{7}$

\section{The lottery example}

b: On Monday all 1000 tickets in a lottery were sold, of which John bought 100 and Bill bought 1 . One ticket was drawn at random on Wednesday.

$e$ : On Tuesday all the lottery tickets except those of John and Bill were destroyed, and on Wednesday one of the remaining tickets was drawn at random.

$h$ : Bill won. (Achinstein 2001: 69).

The claim that Achinstein introduces then is that it is reasonable to make the following assignments of probabilities:

$\mathrm{P}(\mathrm{h} \mid \mathrm{b})=1 / 1000$

$\mathrm{P}(\mathrm{h} \mid \mathrm{e} \& \mathrm{~b})=$ 1/101 (see Achinstein 2001: 69 (with small notational variations here and elsewhere)).

According to Achinstein, independently of what interpretation of probability one adopts (subjective or objective), the positive relevance definition of evidence implies that $e$ is evidence for $h$. This result, however, is implausible according to Achinstein. Here is what Achinstein claims:

Since $\mathrm{P}(\mathrm{h} \mid \mathrm{e} \& \mathrm{~b})>\mathrm{P}(\mathrm{h} \mid \mathrm{b})$, on the positive relevance definition of evidence, $e$ is evidence that $h$, given $b$. But although Bill's probability of winning has increased almost tenfold from 1/1000 to $1 / 101$, do we want to conclude that the destruction of all the tickets except for those of John and Bill is evidence that Bill won? On the positive relevance definition this fact about the ticket destruction is evidence that John won, and it is also evidence that Bill won. Isn't it much more plausible to conclude that this is evidence that John won, not Bill, since on Tuesday, as a result of the ticket destruction, John owned 100 of the 101 remaining tickets? (Achinstein

\footnotetext{
${ }^{6}$ See, for instance: " $e$ confirms or supports $h$ just in case $P(h \mid e)>P(h)$ " (Howson and Urbach 2006: 91-92, original emphasis).

${ }^{7}$ In various places Achinstein provides a number of further similar examples against positive relevance, e.g. a swimming champion entering a pool and thereby raising the probability of his death by drowning, without it being the case that his entering the pool is evidence that he will drown and so on. For the sake of the argument, I will assume that all these examples are sufficiently similar for the purpose of the present discussion.
} 
2001: 70)

The underlying line of thought that Achinstein seems to put forward here is as follows. The positive relevance model predicts that $e$ is evidence in this case for both the proposition $h$ (i.e. Bill won) and for a distinct proposition, say, $h^{*}$ (i.e. John won). Yet this result is implausible, because it is natural to think that $e$ supports $h^{*}$ rather than $h$ (for one thing, $h$ and $h^{*}$ are incompatible, and, presumably, a proposition cannot be evidence for each of two mutually incompatible propositions). According to Achinstein, the positive relevance view is wrong, since it predicts a counter-intuitive result, namely, that $e$ is evidence for $h$.

One way to try to make the intuitive judgement that $e$ is not evidence that $h$ in Achinstein's lottery case more vivid is to focus on our ordinary understanding of functions of the concept of evidence. It is central to our ordinary understanding of evidence that a piece of evidence that $p$ is a reason or good grounds to believe that $p$. However, in the lottery case, $e$ cannot be evidence in favour of the claim that Bill won, because if it were we would have to accept the counter-intuitive claim that something can be both (good) grounds for believing $h$ and (good) grounds for believing not- $h$ (alternatively, something would be a reason for believing $h$ as well as a reason for believing not- $h$ ). For in this case $e$ would be evidence for the claim that Bill won and would also be evidence for the claim that Bill didn't win (since from the claim that John won we can infer that Bill didn't win). This line of thought underlies Achinstein's recent appeal to "Principles of Reasonable Belief". Two of the most relevant of these for our purposes are the following:

The First Principle of Reasonable Belief: If $e$ is evidence that $h$, then $e$ is a good reason to believe h"; and "The Second Principle of Reasonable Belief: If $e$ is a good reason to believe $h$, then it cannot be a good reason to believe a hypothesis incompatible with $h$. (Achinstein 2013: 24)

According to Achinstein, proponents of the positive relevance view who reject his objection from the lottery case face the challenge of showing why these utterly plausible principles should not be accepted as valid after all. See, for instance:

On my view, which is defended in my 2001 [...] what evidence has to supply is simply a good reason for believing, not necessarily a reason that is better, or more convincing, or more confidence-producing than before (though, of course, it can do that too). (Achinstein 2004: $4)$.

The next section introduces two possible replies from the probabilist camp and provides some additional clarificatory remarks on the probabilistic approach to evidence and confirmation. 


\section{Old and new probabilistic strategies}

There are number of things that proponents of the probabilistic approach can say in the light of Achinstein's counter-examples. And numerous replies to Achinstein already exist in the literature (see Bar-Hillel and Margalit 1979, Kronz 1992, Maher 1996, Roush 2004, among others). ${ }^{8}$ Here I present only two lines of thought. The first, a prominent view within the probabilist camp, appeals to an alleged ambiguity of 'evidence'. The second distinguishes comparative claims about being supported by evidence/having evidential support from categorical/positive claims about being evidentially supported/having evidential support. My conclusion will be that the former response is misguided (and hence that Achinstein is right in rejecting it), while the latter is on the right track. However, in order to get there in later sections we have to start with some basics.

Proponents of the probabilistic approach (i.e. those who agree to define evidential support in terms of probability alone) often distinguish incremental evidential support (or incremental confirmation) from absolute evidential support (or absolute confirmation). More precisely, according to this approach, there is a sense of 'evidence $e$ supports a hypothesis $h$ ' according to which (given some background information) $e$ is positively relevant for $h$, where it might still turn out that on balance not- $h$ is better supported than $h$. That a home pregnancy test delivers a negative result (given some background information, such as, for instance, that the test taker is a woman) is incremental evidence that its taker is not pregnant, according to this understanding. Yet, given the high rate of false negatives in home pregnancy tests (and given the background information), the hypothesis that the test taker is not pregnant is not absolutely confirmed by the mere fact that the home test delivered a negative result (more information should be gathered to reach absolute support - a correctly executed blood test at a doctor's office would do the trick). The other sense of 'evidence $e$ supports a hypothesis $h^{\prime}$, then, according to (some) probabilists, is the sense according to which $e$ supports $h$ absolutely. This is a sense according to which (given some background information) evidence $e$ makes hypothesis $h$ highly supported - that is, on balance, evidence for $h$ has reached an important threshold. For example, this is the alleged sense of 'supported by evidence' in sentences like: "That the Earth is not flat is supported by evidence". That the Earth is not flat is absolutely supported by evidence. The idea is that a salient threshold (of support) has been reached for that hypothesis.

A number of prominent philosophers have endorsed the distinction between incremental evidential support/evidence and absolute evidential support/evidence ${ }^{9}$ (see Carnap 1962, Salmon

${ }^{8}$ See also Achinstein 1981, 1992, 1996, 2001, 2004 for his responses to these replies.

9 It is often presented as a distinction between incremental confirmation and absolute confirmation. 
1973, 1983, among others). Moreover, the distinction seems to have acquired the status of orthodoxy within contemporary debates. It is not rare to see that introductory texts on confirmation or evidential support take this distinction to be the standard one. ${ }^{10}$

This distinction then may lead one to think that an appeal to two distinct genuine senses (or concepts) of evidence is the right reaction to Achinstein's cases. This is roughly the main idea underlying the ambiguity response to Achinstein's counterexamples. This line of reply relies in particular on distinctions that have been proposed by Carnap and Salmon. ${ }^{11}$ According to it, not only are there two senses of 'evidence' or 'confirmation' (e.g. an incremental sense (an increase in confirmation, firmness) and an absolute sense (a high confirmation sense)) but, also once we admit the distinction we see that Achinstein's examples don't speak against the positive relevance view. According to the ambiguity response, while positive probabilistic relevance is sufficient and necessary for incremental evidence, it is neither for evidence in the absolute sense. Thus, Achinstein's cases are misguided, according to this line of thought. For if we focus on the incremental sense of 'evidence that $h$ ', then Achinstein's cases are supposedly harmless, since it is still intuitively plausible that there is an increase in confirmation/firmness (incremental evidence) in the lottery case. But if we focus on the absolute sense of 'evidence that $h$ ', then Achinstein's cases are impotent, since there is no evidence in this absolute sense in the lottery examples. In short, Achinstein's cases are irrelevant, according to this line of reply, since absolute evidence was never defined in terms of increase in probability.

10

See, for instance: "Let us say that $E$ raises the probability of $H$ if the probability of $H$ given $E$ is higher than the probability of $H$ not given $E$. According to many confirmation theorists, ' $E$ confirms $H$ ' means that $E$ raises the probability of $H$. This conception of confirmation will be called incremental confirmation. Let us say that $H$ is probable given $E$ if the probability of $H$ given $E$ is above some threshold. (This threshold remains to be specified but is assumed to be at least one half.) According to some confirmation theorists, ' $E$ confirms $H$ ' means that $H$ is probable given $E$. This conception of confirmation will be called absolute confirmation" (Maher 2005: 433). See also Hájek and Joyce (2008) and Kelly (2014), among others.

${ }^{11}$ For instance, Carnap proposes to distinguish two triples of concepts of confirmation/evidence. According to Carnap, on one hand there are concepts that are "concerned with the question of how probable the hypothesis $h$ is on the basis of the evidence $e$ " (Carnap 1962: xv-xvi) and on the other hand there are concepts that "relate to the question as to whether and how much the probability of $h$ is increased when new evidence $i$ is acquired" (Carnap 1962: xvi). Carnap calls the former 'concepts of firmness' and the latter 'concepts of increase in firmness' (cf. Carnap 1962: xvi). And for each of these Carnap defines further distinctions. Namely, both concepts of firmness and concepts of increase in firmness admit, according to Carnap, three further conceptual distinctions: for both of these there are classificatory concepts, comparative concepts, and qualitative concepts. A final complication in Carnap is that among the comparative concepts we can make even further distinctions with respect to what are we comparing. But for our purposes here it suffices to note only the general distinction between concepts of firmness and those of increase in firmness. 
Unsurprisingly, Achinstein rejects the ambiguity response to his counterexamples. According to Achinstein it is an error to suppose that 'evidence' or 'confirmation' is ambiguous in the sense that Carnap and Salmon think it is. Without going into the details of the considerations that Achinstein puts forwards against the ambiguity response (some of which are related to and will be considered in what follows), we can, I think, agree with Achinstein on this point. Indeed, 'evidence' doesn't seem to possess the typical hallmarks of ambiguity. Testing ambiguity may be somewhat tricky, but at least 'evidence' does seem to be clearly different from paradigmatic ambiguous terms, such as 'bank', for instance.

According to Achinstein, the talk about increase in confirmation/evidential strength can be explained by appeal to confirmation/evidence. The former is, in a sense, parasitic on the latter. See, for instance:

Contrary to what probabilists claim, the concept of increase-in-strength-of-evidence (which they attempt to analyze as positive relevance) requires a prior notion of evidence (which they associate with high probability). (Achinstein 2001: 74).

Achinstein agrees to distinguish claims about an increase in evidence from claims about a high degree of evidence, but rightly refuses to admit that 'evidence' is ambiguous:

Finally, is there a distinct sense of "evidence" according to which it means "increase in strength of evidence (or confirmation)," as well as a distinct sense according to which it means "evidence (or confirmation) of a high degree of strength," as Carnap and Salmon claim? No more, I suggest, than there is a distinct "increase-in-degree-of-probability" sense of "probability," an "increase-in-firmness" sense of "firm," or an "increase-in-salary" sense of "salary." In each of these cases, including evidence, we are dealing with something that admits both of increases and of high degrees or amounts. The fact that we can distinguish claims about increases from claims about high degrees does not establish that the concept of evidence (or probability, or firmness, or salary) is ambiguous. (Achinstein 2001: 75).

However, even if we can agree with Achinstein that our ordinary term 'evidence' is not ambiguous in any standard sense (i.e. there are no two senses of 'is evidence that $h$ '), we can still question Achinstein's overall rejection of probabilist approaches. There seems to be another possible reply to Achinstein's argument that a proponent of the idea that an increase in probability has to play a central role in understanding evidence can propose in the light of Achinstein's counterexamples. It is a response that is not an ambiguity response but can avoid Achinstein's conclusions by distinguishing different claims that we can make about evidence/confirmation. The line of response that I have in mind starts by admitting that Achinstein is right on claims about $e$ being evidence that $h$. That is, Achinstein's examples do indeed show that it is not the case that $e$ is evidence that $h$ if and only if the 
probability of $h$ given $e$ is higher than the probability of $h$ without $e$ (i.e. $\mathrm{p}(h \mid e)>\mathrm{p}(h)$ ). For there are cases, like the lottery case, where $\mathrm{p}(h \mid e)>\mathrm{p}(h)$ but $e$ is not evidence that $h$ (if we focus on the ordinary sense of ' $e$ is evidence that $h$ '). But, according to this possible reply, it is still the case that if $\mathrm{p}(h \mid e)>$ $\mathrm{p}(h)$ then $h$ has more evidence/support/confirmation with $e$ than it has without $e$. Or at any rate that if $\mathrm{p}(h \mid e)>\mathrm{p}(h)$, then $h$ is more supported given $e$ than it is without $e$. Surely, such a possible view is not the main focus of Achinstein's argument, as we have seen above (cf. section 2). But this doesn't mean that proponents of the probabilistic relevance approach cannot restrict their proposal to comparative claims about degrees of evidence/support/confirmation. Probabilists who restrict the positive relevance claim only to comparative claims in this way may then avoid Achinstein's counterexamples, since these are formulated as attacks against views that define what it is for something to be evidence that $\mathrm{p}$ (i.e. to evidence claims in the simple positive, categorical sense). This approach is not introducing two concepts or senses of 'evidence', but only distinguishing positive claims (categorical claims) about evidence and confirmation (e.g. claims about a hypothesis $h$ being evidentially supported given $e$ ) from comparative claims (degree claims) about evidence and confirmation (e.g. claims about $h$ being more supported given $e$, than without $e$ ). So, a probabilist who takes this line of response can absolutely agree with Achinstein that in the lottery example, the destruction of all the tickets except for those of John and Bill is not evidence that Bill won, and that 'evidence' here is not ambiguous, but still also maintain that the hypothesis that Bill won has more evidential support (is more supported) given that all of the tickets except for those of John and Bill were destroyed, than it has without the destruction of the tickets. Crucially, of course, probabilists can maintain the probability assignment: the hypothesis that Bill won is more probable given that all of the tickets except for those of John and Bill were destroyed, than it is without it being the case that all tickets except for those of John and Bill were destroyed. For reasons of simplicity let's call such a conciliatory probabilist view the positive probabilistic relevance view about comparative evidential support (PRCE).

One might think that such a move would constitute a way to reconcile our intuitions in Achinstein's cases and a probabilist approach. At least, one might think that there is no incompatibility in accepting Achinstein's claims about the lottery case and endorsing a version of probabilism that doesn't attempt to define being evidence that $h$ in terms of an increase in probability. However, it appears that Achinstein might be committed (at least implicitly) to the denial of such a conciliatory view (that is, to the denial of a PRCE-based strategy). For in his argument against the ambiguity response and in further considerations against other possible replies that we haven't examined (for instance, his rejection of the slight increase in evidence response; see Achinstein 2001: 75-76), Achinstein commits himself to the idea that evidence is a 'threshold' concept and as such 
requires that a certain amount of probability be reached for $h$, in order for it to be the case that $h$ has more evidence, evidential support, confirmation (or firmness for that matter) with $e$ than $h$ has without $e$ ('threshold claim'). This idea, however, seems to be incompatible with the claim that endorsing PRCE (and giving up the simple relevance view about conditions for evidence that $h$ ) is enough for a probabilist to avoid Achinstein's criticism. Consider, for instance, the following quotation from Achinstein:

In the light of these three examples perhaps it will be agreed that 'e is evidence that h' cannot be defined simply as 'e increases h's probability'. But it may be contended that a related concept can be so defined, viz. 'e increases the evidence that h'. Thus e increases the evidence that $h$ if and only if $p(h, e \& b)>p(h, b)$. However, increasing the evidence that $h$ is not the same as increasing the probability of $h$. To increase the evidence that $h$ is to start with information which is evidence that $h$ and add to it something which is also evidence that $h$ or at least is so when conjoined with previous information. But to do this it is neither sufficient nor necessary to increase h's probability. The first lottery example shows that it is not sufficient [...]. In the first lottery example there is no increase in evidence that Bill will win, since in the first place there is no evidence that he will win, and the combined new and old information is not evidence that he will win, even though the probability that he will win has increased. (Achinstein 1983: 153, emphasis added).

As I understand this passage (and similar passages in later works), Achinstein suggests here that in order for something to increase evidence for a hypothesis, the hypothesis has to have (some) evidence in its favour in the first place. In other words, in order for $h$ to receive an increase in evidence/support/confirmation from $e, h$ must already have some evidence/support/confirmation. Of course, PRCE doesn't require this. PRCE only suggests that if something increases the probability of a hypothesis then it also increases evidence in its favour (it has more support/confirmation with $e$ than it has without $e$ ). PRCE doesn't add the further condition that this can happen only if the hypothesis has (some) evidential support.

In defending the 'threshold claim' about evidence Achinstein also suggests that concepts to which the concept of evidential support has been tied (or with which it is associated) - e.g. concepts of acceptability, firmness, confident belief, founded belief - are all threshold concepts. Evidential support, according to Achinstein, is like these other threshold concepts. According to Achinstein, there is a specific salient threshold of $X$, such that exceeding X makes a hypothesis firm, supported, 
a founded belief, etc. ${ }^{12}$ However, this specific threshold has not been attained in the lottery case, according to Achinstein. Hence, it is not appropriate to talk of acceptability, foundation, support, or firmness in this case at all. According to this line of thought, $e$ is not evidence for $h$ in the lottery case, even though there is a slight probability increase of $h$, for the salient threshold for acceptability, support, foundation, or firmness has not been reached. In short, our intuitive judgement about this case is not due to some framing effect, but follows from our common-sense understanding of notions like support, acceptability, and so on. See, for instance:

Again I invoke the idea of threshold concepts. According to Carnap, Salmon, and Maher, the present concept of evidence is tied to the idea of "making $h$ firmer or more acceptable," "making it rational to be more confident that $h$ is true," "giving $\boldsymbol{h}$ more support." But if $e$ makes $h$ firmer (more acceptable, etc.) than it was without $e$, then, with $e, h$ has at least some firmness. But firmness, and the other concepts invoked, are threshold concepts with respect to probability. It is necessary that some threshold of probability be exceeded if a hypothesis is to have any firmness at all.

The fact that I am now riding on an elevator increases the risk of my being injured in an elevator accident from 0 to 1 in 6 million. [...] But it gives no firmness (acceptability) at all to that hypothesis. The reason it seems (and is) implausible to speak of evidence in such a case has nothing to do with the fact that the boost in probability is so small. The reason is rather that the probability itself is so small. (Achinstein 2001: 75-76, emphasis added). ${ }^{1314}$

The point I would like to make in the rest of this paper is that this further claim, i.e. the "threshold claim', from Achinstein is misguided. Once we see this point, we will be in a position to both fully appreciate and, indeed, pay due respect to Achinstein's cases, and also respect the general probabilist intuition that an increase in probability matters for evidential support (by accepting PRCE). Hopefully, this will then constitute a way out of the deadlock facing proponents of Achinstein's counterexamples and probabilists. Before that, however, let us proceed by reconstructing in detail

12 See Achinstein: "A necessary condition that must be satisfied for hypothesis $h$ to have any acceptability, foundation, or firmness, and before I have any confidence in it, is that $h$ 's probability exceed some threshold" (Achinstein 2001: 73-74).

$13 \quad$ See also: "There is not enough probability here to exceed a threshold necessary for me to have any confidence (for the hypothesis to have any firmness, support, etc.)" (Achinstein 2001: 74, emphasis added).

${ }^{14}$ As I am reading this passage I interpret "etc." and "the other concepts invoked" above as including a reference to the concept of being supported. 
what we can reasonably take to be an implicit argument from Achinstein against the PRCE strategy.

\section{What would Achinstein reply?}

What I have called 'the PRCE strategy' is the view that combines: (i) the judgement that Achinstein's cases are genuine counterexamples against the view according to which $e$ is evidence that $h$ if and only if the probability of $h$ given $e$ is higher than the probability of $h$ without $e$ (i.e. $\mathrm{p}(h \mid e)>\mathrm{p}(h)$ ), and (ii) the claim that if $\mathrm{p}(h \mid e)>\mathrm{p}(h)$, then $h$ has more support/evidence/confirmation given $e$ than it has without $e$. I have assumed that Achinstein rejects (implicitly, at any rate) the PRCE strategy. Some of the considerations that he has put forward against possible probabilist replies to his counterexamples seem to commit him to the denial of (ii). In this section I will reconstruct these considerations in a more explicit way and isolate a specific, implicit premise that I believe is wrong. The aim of the next section will be to provide a new argument, inspired by recent work on gradable adjectives, to show what exactly is wrong with this implicit premise in Achinstein's considerations against possible probabilist replies. Two remarks are in order before the reconstruction of the argument.

First, if, at the end of the day, I am proven to be wrong in assuming that Achinstein is implicitly committed to denial of (ii) and hence, nothing in his writings warrants us to think that he will reject the PRCE strategy, then I will only be happy with such a conclusion and will consider that my task has been accomplished - namely, the task of clarifying the debate and bringing the deadlock in the debate between Achinstein and the probabilists towards an end. However, I also think that for the reasons stated above, we are entitled to assume that Achinstein is implicitly committed to the denial of (ii).

Second, my reconstruction of Achinstein's argumentative considerations and my own positive argument rely on some assumptions about some rough equivalences of 'gives support', 'has support' and 'is supported'. More precisely, I will assume (A1) that phrases like ' $e$ gives $h$ support' and ' $h$ has support/evidential support/confirmation given $e$ ' can be paraphrased without any significant (for our purposes) loss in meaning by phrases such as ' $h$ is supported/confirmed/evidentially supported by $e$, and (A2) that phrases like ' $e$ gives greater support to $h$ than the support that $h$ has without $e$ ' can be equally paraphrased as ' $h$ has more support given $e$ than it has without $e$ ' and ' $h$ is more supported given $e$ than it is without $e^{\prime}$. Again, if I am proven wrong on this, i.e. about these equivalences, and the arguments to follow are not valid if, for example, instead of 'being more supported' the premises contain 'giving greater support' or 'having more support', then we are back to the idea that there need not be any tension between accepting Achinstein's cases and maintaining a revised version of the 
probabilistic relevance approach (e.g. a PRCE strategy).

For the sake of simplicity, let us refer to (a simplified version of) (ii) the PRCE strategy (positive probabilistic relevance view about comparative evidential support) in the rest of this and the next section by the following precisification of 'PRCE':

(PRCE) For all relevant $h$, and all relevant $e$, if $\mathrm{p}(h \mid e)>\mathrm{p}(h)$, then $h$ is more supported given $e$ than it is without $e^{15}$

PRCE was introduced as a possible way to escape Achinstein's counterexamples. However, I do not think it is alien to most versions of the positive relevance approach. I think it presents a natural retreat for proponents of the ambiguity approach to 'evidence' and positive relevance theorists more generally (e.g. Carnap 1962: 462, Salmon 1983, Howson and Urbach 2006: 91-92). If one thinks that the pregnancy test example speaks in favour of 'incremental evidence' being defined in terms of an increase in probability, then one should, I think, also take it that it speaks in favour of PRCE. A home test indicates that you are pregnant. One might think that, given some background information, this fact increases evidential support for the hypothesis that you are pregnant. Crucially, that you are pregnant given the positive home test result (and some background information) has a higher probability than that you are pregnant without the test result being positive (given the background information). Consider a case where the data that were obtained through a well-designed statistical study raise the probability that asbestos is a cancer risk by $15 \%$. According to this view, if the probability of the hypothesis that asbestos is a cancer risk is raised by $15 \%$ by the results of the study, then that study increases support for the claim that asbestos is a cancer risk and the cancer risk hypothesis is more supported given this data than without it. Hence, one might think that such (and many similar) cases speak in favour of PRCE.

As we have seen above, Achinstein's considerations against possible responses to his counterexamples also appear to contain an implicit argument against PRCE. This argument relies on two general principles. First, it relies on the claim that an increase in firmness, support, acceptability, and so on, of a hypothesis given a piece of evidence, entails that the hypothesis is firm, supported, acceptable, and so on, given the piece of evidence. In short, that a hypothesis has more support given

${ }^{15}$ Which $h$ and $e$ count as relevant is left out of discussion here. This qualification is intended to deal with the fact that according to many, including Achinstein and Maher, a priori or necessary truth will not count as evidence. What exactly this amounts to is not the main object of our discussion. However, we might want to respect the idea that only empirical propositions can count as evidence. 
a piece of evidence entails that the hypothesis is supported given the piece of evidence in question. Let us call this the bridge principle. Second, it relies on the claim according to which if a hypothesis is firm, supported, acceptable, and so on, given a piece of evidence, then its probability given that piece of evidence has reached some relevant threshold $x$. Being evidentially supported entails having a high enough probability given the relevant piece of evidence. In other terms, it cannot be that a hypothesis is firm, acceptable, etc. and that its probability (given the relevant evidence) is below some salient threshold of probability. Let us call this claim the probabilistic threshold principle. The thought then is that in the relevant cases (e.g. the lottery case, the elevator case), the probability of the hypothesis given the relevant proposition is so small that the hypothesis is not firm, acceptable, or supported. Since in these cases, the hypothesis is not supported, given the relevant proposition, it follows, according to Achinstein, that the hypothesis is not more supported given the relevant proposition than it is without it.

With these remarks in mind, we are now in a position to propose a possible reconstruction of Achinstein's implicit argument against PRCE in the form of the following schema:

\section{((Reconstruction of) Achinstein's argument from cases of improbable hypotheses)}

(1) For any relevant hypothesis $h$, for any relevant proposition $e$, if the hypothesis $h$ is more supported given $e$ than it is without $e$, then $h$ is evidentially supported given $e$. [Bridge Principle]

(2) For any relevant hypothesis $h$, if the probability of $h$ given a piece of evidence $e$ doesn't exceed the relevant threshold $x$ of high enough probability, then $h$ is not evidentially supported given $e$. [Probabilistic Threshold Principle]

(3) The probability of some hypothesis $h$ for $S$ given $e$ doesn't exceed the relevant threshold of high enough probability $x$. [Cases include the elevator accident, the lottery case 'Bill won']

(4) The hypothesis $h$ is not evidentially supported for $S$, given $e$. [2, 3, MP]

(5) It is not the case that the hypothesis $h$ is more supported given $e$ than it is without e. $[1,4, \mathrm{MT}]$

(6) The hypothesis $h$ has a higher probability given $e$ than it has without $e$. [Cases include the elevator accident, the lottery case].

(7) For all relevant $h$, and all relevant $e$, if $\mathrm{p}(h \mid e)>\mathrm{p}(h)$, then $h$ is more supported given $e$ than it is without $e$ [PRCE, for the sake of reductio]

(8) The hypothesis $h$ is more supported given $e$ than it is without $e$. [6, 7, MP] 
Premises (1)-(8) together lead to a contradiction. They are mutually inconsistent. According to this possible argument from Achinstein, this would show that PRCE cannot be true. As we saw above, there are some reasons for thinking that Achinstein is at least implicitly committed to all premises except (7), PRCE. According to my understanding of Achinstein's considerations against the probabilist approach, his views are incompatible with PRCE. If all other premises are more plausible than premise (7), then the rational thing to do is to give up PRCE. Note again that if, contrary to appearances, Achinstein is not committed to one or another of the other premises (that is, any premise apart from 7), then it is not clear on what basis Achinstein could reject (7) after all.

\section{Linguistics of gradable adjectives and a problem for Achinstein's account of 'supported'}

The argument from the Bridge and Probabilistic Threshold principles may appear appealing. And yet it is also problematic. Premise (1) of this argument is mistaken, or so I will argue in this section.

Premise (1) is entailed by the Bridge principle applied specifically to an increase in support:

\section{(Bridge Principle connecting comparative to categorical support (Bridge Support))}

For any hypothesis $h$, for any proposition $e$, if the hypothesis $h$ is more supported given $e$ than it is without $e$, then $h$ is evidentially supported given $e$.

As we saw above, for Achinstein this claim is part of an even more general principle according to which having more firmness, acceptability, rational confidence, support, and foundation, given a proposition, entails having firmness, acceptability, rational confidence, support, and foundation, given the proposition in question. Achinstein appears to suggest that this principle holds because the relevant concepts (firmness, acceptability, foundation, support, and so on) are what he calls 'threshold concepts'. In sum, Achinstein's implicit argument relies crucially on some simple linguistic and allegedly common-sense considerations about our ordinary understanding of support. According to this line of thought, our very concept of support, that is, our (ordinary) understanding of the term support (and of related terms) is such that the term supported validates the pattern captured in (Bridge Support).

Although Achinstein is right that supported and the like don't behave quite like more stereotypical gradable adjectives, a more attentive and linguistically informed inspection of the relevant terms shows that the (Bridge Support) principle ultimately doesn't hold. ${ }^{16}$ In short, if 
(Bridge Support) and our theoretical considerations about evidential support in general have to respect our ordinary language use, then, given the insights from our best contemporary theories of gradable adjectives, we can conclude that in some situations it is possible both for a hypothesis to be more supported given the relevant evidence than it is without it, and still not to be supported tout court. Linguistic insights suggest that the pattern captured in (Bridge Support) is not always applicable for the gradable adjective supported and the like. The mere fact that supported is, in a sense, a threshold term doesn't guarantee that it always respects the relevant pattern. The remainder of this section provides a brief exposition of the relevant aspects of the linguistic considerations about gradable adjectives in general, as well as an argument inspired by those considerations that shows that the case of supported and the like is more complex than Achinstein's (possible) argument presupposes. ${ }^{17}$

three-year-old child, it doesn't follow at all that the five-year-old child is tall (she may well be short for her age). And from the fact that to kill a victim painlessly is better than to kill the victim painfully, it doesn't follow at all that to kill a victim can be good.

${ }^{17}$ At this point, one might object that even if there is a natural use of supported in our ordinary language that doesn't validate the Bridge Support principle, we are still not entitled to give up the Bridge Support principle. The thought here would be that the conciliatory position (see below for details) according to which, roughly, one could provide a probabilistic account of comparative support (e.g., ' $\mathrm{x}$ is more supported than $\mathrm{y}$ ') but not of categorical support (e.g., 'x is supported') is ultimately unwarranted, because it is misleading. In some cases where a hypothesis (e.g., 'I will win the lottery') is not supported (i.e. 'that I will win is supported' is false), the conciliatory view still implies that the hypothesis is comparatively supported (e.g., it is true that 'the hypothesis that I will win is more supported, given that I've bought a ticket in a 1 million lottery, than it is without my having bought a ticket'). But providing a claim about there being comparative support in cases where there is no categorical support might be misleading and counterintuitive in many scientific and nonscientific contexts. There are two things that I would like to propose in reply to this worry. First, I acknowledge that it might indeed be uncooperative and misleading to reply that the notion that one will win the lottery is more supported now that one has a ticket compared to when one didn't even have a ticket, when one is asked whether the claim that one will win the lottery is supported. Of course, the same would go for any other case of a similar structure (although I would also like to point out that there might be situations where providing the comparative claim is not misleading, for instance, when historians of science are investigating which one of two competing outdated theories was more supported). But this aspect can be perfectly explained by appeal to Gricean maxims of communication without accepting the Bridge Support principle (by appeal to the maxim of relation/relevance, for instance, cf. Grice 1975). Strictly speaking, comparative claims about being supported can be true without corresponding categorial claims being true. Second, I would like to note that this line of defense of the Bridge Support principle appeals to something beyond our common-sense judgements and ordinary language use. Namely, it questions the theoretical utility of claims about comparative support. The part of Achinstein's argument that we are focusing on here is his appeal to our ordinary understanding of support, firmness and so on. Hence, unfortunately, a full reply to this challenge lies beyond the scope of the present article. I would like to thank an anonymous reviewer for this journal for making me aware of this potential worry. 


\subsection{Varieties of gradable adjectives}

Gradable adjectives can be distinguished from non-gradable adjectives by means of some relatively simple tests involving comparative constructions, comparative questions, and constructions with degree modifiers (cf. Kennedy 2007). See, for instance, examples in (1)-(3):

(1) The Amazon rainforest is larger than Texas. [A comparative construction]

(2) How tall is this wall? [A comparative question]

(3) The floor is completely dry. [A construction with a degree modifier]

Only gradable adjectives combine felicitously in these sorts of constructions (that is, in standard contexts). Compare these to some typical cases of non-gradable adjectives in (4)-(6):

(4) ? This bottle is more plastic than that one. ${ }^{18}$

(5) ? How next is this championship?

(6) ? She is slightly/completely married.

Crucially, not all gradable adjectives are alike. According to a recently popular approach, there are relative gradable adjectives on the one hand, and absolute gradable adjectives on the other hand (see Kennedy and McNally 2005, Kennedy 1999, 2007, see also Unger 1975, and Rotstein and Winter 2004). Very roughly, a fundamental difference between the two is that the former are utterly context-sensitive, whereas the latter are less context-sensitive and have an inherent standard of application (that allows nevertheless for some precisification in a context). ${ }^{19}$ Perhaps the two most notable differences in the behaviour of these two concern their combination with certain degree modifiers and their behaviour with respect to certain patterns of inferences among antonyms. Consider, for instance, examples in (7)-(10):

(7) ? This wall is slightly/minimally/completely/totally/absolutely/100\% tall/short.

(8a) The sidewalk is completely/totally/absolutely dry.

(8b) The floor is slightly wet.

(9a) Oscar is not tall.

(9b) ? Oscar is short. [The observation here is that one cannot felicitously infer (9b) merely on the grounds of the truth of (9a)]

(10a) The sidewalk is not dry.

19 There is a sense, I think, in which what Achinstein calls 'threshold' concepts might actually correspond to the broad category of what linguists call 'absolute gradable adjectives'. However, we lack textual grounds to claim this with any certainty. 
(10b) The sidewalk is wet. [The observation here is that it seems OK to infer (10b) merely given (10a)]

The adjectives 'tall', 'short', and the like (i.e. relative gradable adjectives) don't combine well with the relevant degree modifiers, which seem to be dedicated to gradable adjectives of a different sort (i.e. absolute gradable adjectives). The inference patterns in (9a)-(9b) and (10a)-(10b) demonstrate that while there is an acceptable inference from the mere fact that something is not dry to the fact that the thing is wet, nothing like this sort of inference holds among antonyms with respect to adjectives like 'tall', 'short', and the like. The inference pattern among antonyms seems to be validated only in the case of absolute gradable adjectives and not in the case of relative ones.

Another recently much discussed observation is that adjectives within the absolute gradable adjective group can be further distinguished. Consider the following (perhaps the two most notable) differences in behaviour within the group of absolute adjectives. The first concerns combination with degree modifiers, and the second concerns patterns of inferences: ${ }^{20}$

(11a) This sample of water is completely pure.

(11a)? This sample of water is slightly pure.

(12a)? Water in Chernobyl is completely impure.

(12b) Water in Fukushima is slightly impure.

(13a) The window is completely opaque.

(13b) The window is slightly opaque.

Certain absolute gradable adjectives combine well with degree modifiers like 'slightly' but not with degree modifiers of the 'completely' sort. Other absolute gradable adjectives combine with 'completely' and the like, but not with 'slightly'. Still others may be combined with both sorts of degree modifiers.

(14a) Chernobyl is more impure than Fukushima.

(14b) Chernobyl is impure.

(15a) Fukushima is purer than Chernobyl.

(15b) Chernobyl is not pure.

Certain absolute gradable adjectives respect the inference pattern in (14a)-(14b), while others conform to the pattern exhibited in (15a)-(15b). That is, certain adjectives are such that if we hold (14a) (or a similar proposition) to be true, then (14b) (or a similar proposition) appears to follow. adjectives are to be taken with a grain of salt. It is not claimed that all gradable adjectives in all contexts will validate this sort of behaviour. These are patterns of typical behaviour in normal contexts. 
And other adjectives are such that if we hold (15a), then (15b) follows. Importantly, typically absolute adjectives respect either one or the other of these patterns. It doesn't seem OK to infer that Fukushima is not impure from (14a) alone. And it doesn't seem OK to infer that Fukushima is pure from (15a) alone. I say 'typically' because there is a third category of absolute gradable adjectives that appear to allow, in some contexts, for inferences of the (14a)-(14b) sort and in other contexts for inferences of the (15a)-(15b) sort. Perhaps 'full', 'transparent', and 'opaque' are good examples of this third sort (see McNally and Kennedy 2005, and Kennedy 2007, for cases that appear to warrant this observation).

Absolute adjectives that only combine well with completely and the like, and conform to the (15a)-(15b) style pattern of inference (but not the (14a)-(14b) pattern), are called within the Kennedy and McNally approach (Kennedy and McNally 2007, Kennedy 1999, 2007) maximal standard absolute gradable adjectives. The inherent standard that is required in order for something to be pure, dry, and so on, is fixed in terms of having the maximal amount of the relevant property (e.g. pureness, dryness, and so on). Adjectives that only combine with slightly and the like, and respect the (14a)-(14b) style pattern of inference (but not the (15a)-(15b) style pattern), are called within the Kennedy and McNally approach minimal standard absolute gradable adjectives. The inherent standard that is required in order for something to be impure, wet, and so on, is fixed in terms of having any amount whatsoever of the relevant property (e.g. impurity, wetness, and so on) - it only has to satisfy a minimal standard. Adjectives that appear to behave in some contexts like maximal standard adjectives and in other contexts as minimal standard adjectives are called maximal and minimal standard absolute gradable adjectives.

The theory behind this classification (i.e. the Kennedy and McNally framework) appeals to the well-known and popular idea that gradable adjectives should be understood by appeal to degrees and their underlying scales (see Bartsch and Vennemann 1972, Cresswell 1976, Heim 1985, and others). According to Kennedy and McNally (see Kennedy 1999, 2007, Kennedy and McNally 2005), a gradable adjective is a measure function that takes an individual (or an entity) as an input and maps it to a degree on the relevant scale as an output. ${ }^{21}$ This account of gradable adjectives appeals to degrees on scales and also to relations (orderings) that hold among these degrees. Both the comparative form (i.e. ' $x$ is more $a$ than $y$ is') and the positive form of a gradable adjective (i.e. (cf. Kennedy and McNally 2005: 349): $\llbracket$ tall $\rrbracket=\lambda d \lambda x$.tall $(x)=d$, where tall is a measure function that takes an individual, $x$, as an input and gives $x$ 's degree of tallness as the output. The degree of tallness here can be understood as a degree on the scale of vertical extension. Measure functions have the type $<e, d>$; they take individuals (in input) and provide degrees (in output). 
' $x$ is $a$ ') are then explained within this framework by an appeal to orderings among degrees. In the comparative case, something is, say, taller than something else, just in case the degree of the first element on the scale of vertical extension is strictly above the degree of vertical extension of the second element. In the case of the positive form, for instance, to say that someone is tall is to say that the degree corresponding to this person on the scale of vertical extension exceeds the degree of some, presumably, contextually determined standard of tallness. (In the case of absolute gradable adjectives, the relevant standard is determined not in an utterly context-sensitive way, but by an appeal to some inherent standard - a minimal or maximal endpoint on the relevant scale.) Within this approach all gradable adjectives are explained by an appeal to scales and relations among degrees on the relevant scales.

One aspect of Kennedy and McNally's view that appears particularly insightful and novel (in the context of the general scalar approaches) is their focus on mathematical properties of various possible scales in their explanation of gradable terms. In short, their insight is that focusing on possible abstract differences in scale structure may help us explain the differences among gradable adjectives. ${ }^{22}$ Some scales are totally open-ended. They have neither upper nor lower limits. Some are partially open (or, in other terms, partially closed). Partially open scales are of two sorts: some are closed on the upper end but open on the lower part; some have lower limits but no limits on the upper part. Very roughly then, on their view, relative gradable adjectives are modelled as having totally open underlying scales (of the relevant properties). Absolute gradable adjectives have partially or totally closed underlying scales. More specifically, maximal standard absolute gradable adjectives have scales that have an upper endpoint, but are open on the lower part. Minimal standard absolute gradable adjectives are closed on the lower side, but are open on the upper side. And minimal-maximal absolute adjectives have both lower and upper endpoints on their underlying scales. This is a very general and necessarily brief presentation of a popular contemporary theory and classification of various gradable adjectives. Let us turn now more directly to consideration of the semantic properties of 'supported'.

\subsection{The gradability of 'supported' and why Achinstein's argument fails} McNally (2005) note that the basic topological properties of scales can offer some explanations of semantic properties of adjectives. For instance, scales can be open or closed. This provides a typology of adjective meanings, which helps explain some of their interesting semantic properties" (Glanzberg 2014: 274). 
This subsection aims to apply the powerful theory and classification of gradable adjectives (introduced above) to the case of 'supported' and to evaluate Achinstein's argument in the light of this classification. In this respect, first, it seems plausible that supported is gradable. It may be used in comparative constructions and in constructions with degree modifiers; see (16)-(17): ${ }^{23}$

(16) Einstein's theory of relativity is more supported than Aristotelian physics is. ${ }^{24}$

(17) That the Earth is an oblate spheroid is totally/absolutely/completely supported.

Second, supported appears to be an absolute gradable adjective rather than a relative one. For one thing, it combines well with degree modifiers like completely, fully, totally, absolutely, that are proper to absolute gradable adjectives and it validates entailment with antonyms; see:

(18a) This theory is not supported.

(18b) This theory is unsupported/baseless.

It appears that (18b) follows from (18a), which is a pattern proper to absolute gradable adjectives.

Third, we can make our understanding of supported more precise by classifying it as one of the three possible sorts of absolute gradable adjectives. This task, however, is a slightly more complex one.

For one thing, supported clearly combines with degree modifiers that are typically combined with maximal standard absolute gradable adjectives (completely, totally, etc.; see (17)) and it has poor results in combination with degree modifiers proper to minimal standard absolute gradable adjectives (e.g. slightly); see (19):

(19) ? This theory is slightly supported. ${ }^{25}$

23 Note though that it provides some mitigated results in comparative questions. According to native English language speakers that the author has consulted on this point, 'How supported is this view?' sounds clearly odd. This is somewhat confirmed by poor results from Google searches for expressions like 'How supported is the idea/thought/view'.

${ }^{24}$ According to a referee for this journal and another native English speaker, the 'more than' constructions (e.g. 16) are a bit awkward. Other native English speakers that the author has consulted on this found (16) to be fine. Note also that (at least some) comparative constructions with 'unsupported' appear to be fine. 'The 9/11 conspiracy theory is as unsupported as the Loch Ness Monster Myth' is felicitous. One line of thought suggested by the referee here is that there might be a general worry about tests for the gradability of 'supported' given that 'supported' is a participle. This might explain some of the specific behaviour of 'supported' compared to other (non participle) gradable adjectives. See also footnote 25 for a related line of thought. Unfortunately, a proper treatment of this question goes beyond the scope of the present discussion. Nevertheless, I would like to stress here that typically non-gradable adjectives pass none of the relevant tests for gradability. Hence, as long as we have some data speaking in favour of the gradability of the adjective 'supported', we may reasonably take it to be gradable. Thanks to the referee for pointing out this aspect of tests with 'supported' and to Edgar Phillips for a very useful discussion on this. ${ }^{25}$ An anonymous referee for this journal observes that while (19) is odd, a parallel claim with the scalar noun 'support' seems fine. See, for instance: (a) 'This theory has (only) slight support'. It is 
Moreover, the fact that, in simple cases, supported appears to respect patterns of inferences that are typical for maximal standard absolute gradable adjectives also seems to suggest that the maximal absolute interpretation might be the correct one. See:

(20a) This new theory is more supported than that old one.

(20b) That old theory is not supported.

It appears that (20b) has to follow, given the truth of (20a). This is exactly the pattern of inference that we would expect to go through if supported is a maximal standard absolute gradable adjective. If supported is a maximal standard adjective, then it is to be expected that when a claim is supported, it is maximally supported and, hence, any other claim that is less supported than the given claim will not be supported (since being supported tout court requires a maximal amount of support).

However, one might think that a closer look at the behaviour of supported shows that it doesn't always behave like a maximal only adjective. One might think, for instance, that the pattern captured in (20a)-(20b) is not always a natural one for supported. Consider, for instance, (21a)(21b):

(21a) That it will rain tomorrow given today's weather forecast is more supported than that it will rain after tomorrow.

(21b) ? That it will rain after tomorrow is not supported (given today's weather forecast). "?" in (21b) is supposed to indicate that it doesn't appear intuitive to infer (21b) on the basis of

reasonable to think that the scalar noun 'support' would share the same scale structure as 'supported'. Thus, the difference in our intuitions about (19) and (a) here requires some extra explanation. One quick response is to observe that this oddity seems not to be reserved to 'supported' alone. Other paradigmatic absolute adjectives seem to have a similar problem. It does seem odd to say 'This sample is slightly pure'. However, apparently, 'Our uranium enrichment will not be limited to 3.67 percent of purity' (see http://www.xinhuanet.com/english/201907/03/c_138195642.htm) is fine (which, I take it, implies that true ascriptions of 'purity' to an item doesn't require the attribution of maximal purity to the item in question). Compare also 'His mouth was minimally dry' to 'His mouth had some dryness', where the former seems odd, but the latter appears to be fine. Thus, whatever is going on here, it might be a general worry about how to properly connect scalar nouns to (some) gradable adjectives within the scalar approaches to gradable adjectives. Another more constructive line of reply would be to point towards the specificities of gradable adjectives that are participles. Thus, the specificities of 'supported' (and the noun 'support') and the like might be explained by an appeal to specificities of the event structure of the counterpart verb. How exactly such an explanation would go is beyond our present discussion. The discussion in Kennedy (2007: 36-40) and in Kennedy and McNally (2005) on deverbal adjectives may contain some useful suggestions for further work on this issue. Unfortunately, a fully satisfactory treatment of this question cannot be achieved within the limits of the present article and has to be left for another occasion. Thanks to the referee for making me aware of this point. 
(21a). It may well be the case that our weather forecast service is the best in the world and yet the reliability of its predictions varies with the distance in time: it is most reliable about near future events. In this case, it doesn't appear that we are appealing to some special, non-typical use of supported. One may think that this then undermines our above consideration about supported typically respecting inference patterns proper to maximal standard adjectives, since the example in (21a)-(21b) is a violation of an inference pattern that is proper for absolute maximal standard adjectives.

On reflection, though, it may still be reasonable to maintain that 'supported' is a maximal only adjective. For one thing, the above observed pattern may be explainable by an appeal to some pragmatic factors. Kennedy and McNally, for instance, allow that even absolute gradable adjectives might be somewhat dependent on a given context, though not in the same way as relative ones. While relative ones rely essentially on a context for setting the relevant standard and absolute adjectives have inherent standards, the absolute adjectives may still allow for precisifications in a given context. In other words, while they may not be vague (contrary to relative ones), absolute adjectives may still be imprecise. Thus, in one context it may well be true that the theatre is empty when there are only one or two spectators in it, in another context it may well be false that the theatre is empty when there are only one or two spectators in it. For example, in a context where a director of a theatre is complaining about low attendance at the theatre it will be true that the theatre was empty, while in a context where a police investigator is investigating a murder in the theatre it will be false that the theatre was empty (see Kennedy 2007 and Kennedy and McNally 2005). One line of thought then is that we may still classify 'supported' as a maximal adjective, since it is not clear that the context across (21a) and (21b) has been held fixed and, hence, that no pragmatic factors are responsible for our intuitions about the utterance (21b). Maybe it is the focus on weather predictions about 'after tomorrow' which is responsible for our intuitions, since we may find it in general difficult to predict the weather correctly two days in advance. Furthermore, if we are going to classify 'supported' as both a maximal and minimal standard adjective, we will also need to find a perfectly fine and standard case where inferences proper to minimal standard adjectives hold. However, it is not clear that we can find such inferences. From that it will rain tomorrow given today's weather forecast is more supported than that it will rain after tomorrow given the forecast, we are not, it seems, warranted to infer that that it will rain tomorrow given today's forecast is supported. It may well be that we have good reasons to doubt the reliability of today's weather forecast but still recognize that it may be somewhat more reliable with respect to tomorrow than with respect to after tomorrow. However, if there were a minimal reading of 'supported' then we should be able to recover cases where this sort of inference goes through. It is unclear that we can. 
Finally, even if after all we are wrong on this and 'supported' can be classified as both a minimal and maximal absolute adjective, this will still not affect our overall dialectic. As we will see shortly, as long as 'supported' is not classified as a minimal only adjective, we have good grounds to reject premise (1) in the above argument. ${ }^{26}$

On the basis of these considerations, I would like to suggest that supported is a maximal standard absolute gradable adjective. That is, it is an adjective with a partially closed underlying scale with an upper limit. Supported requires the maximal amount of support in order for a claim to be supported (see the example in (20a) and (20b)).

Now, the problem with Achinstein's argument is that his premise (1) - the principle (Bridge Support) - cannot be true given our conclusions about the nature of the gradable term 'supported'. The (Bridge Support) principle states that it is the case for any hypothesis and for any potential piece of evidence that if a hypothesis is more supported given that evidence than it is without it, then the hypothesis is supported. If this principle were universally valid, then the adjective 'supported' would always behave like a minimal standard absolute gradable adjective only. For the pattern of inference that is captured in (Bridge Support) corresponds in a sense to the pattern that we observe in the behaviour of minimal standard adjectives (see (14a)-(14b)). In the case of minimal standard adjectives, we intuitively judge that the claim with the relevant adjective in positive form follows from the construction with the adjective in comparative form. If an $x$ is more impure/wetter/etc. than a $y$, then we can conclude that the $x$ is impure, wet, and so on. The particularity of minimal standard adjectives is that in order for the claim of the form "an $x$ is $a$ " (where $x$ is a noun and $a$ a minimal standard adjective) to be true, it is required that the object/individual in question has at least the minimal amount of the relevant property. Other gradable adjectives don't validate this sort of behaviour. For instance, maximal standard adjectives violate this pattern. We observed above that the adjective 'supported' falls into the group of adjectives that behaves like a maximal standard adjective. Hence, 'supported' will not respect the minimal standard pattern and, by consequence, will not respect the (Bridge Support) principle. Note that here we are not merely rejecting the (Bridge Support) principle; we are suggesting that given a well-established contemporary theory of gradable adjectives we have theoretical grounds for why 'supported' cannot respect the (Bridge Support) principle. In sum, Achinstein's implicit argument relies on the misleading thought that 'supported' is only a minimal standard absolute gradable adjective.

${ }^{26}$ Thanks to an anonymous referee and Fabrice Teroni for very useful remarks and suggestions on the last two paragraphs. 


\section{Conclusion}

In this paper I have revisited one venerable debate between probabilists and anti-probabilists about evidential support. More specifically, I have reconsidered arguments from one prominent antiprobabilist about evidential support, namely, Peter Achinstein, who has for a long time and on many occasions claimed that probabilistic models face fatal counterexamples. The core of Achinstein's argument is a claim about our ordinary concept of support. A central part of this paper was to clarify what exactly his argument is and whether someone sympathetic to the idea that an increase in probability plays some role in defining some aspects of evidential support may defend a somewhat restricted probabilistic account. My overall proposal is that a conciliatory position can be defended namely, a view that gives full justice to our intuitions about Achinstein's cases and maintains that if $\mathrm{p}(h \mid e)>\mathrm{p}(h)$, then $h$ is more supported given $e$ than it is without $e$. I also examined what appears to be an implicit argument in Achinstein's texts against this new conciliatory proposal. Some of the considerations that Achinstein puts forward against possible responses to his counterexamples appear to contain an argument that appeals to a bridge principle connecting considerations about comparative claims about being supported and categorical claims about being supported - namely, the principle according to which if the hypothesis $h$ is more supported given $e$ than it is without $e$, then $h$ is evidentially supported given $e$. Finally, an important aspect of my article was to assess the plausibility of such a bridge principle. I proposed to assess this principle in the light of recent work on the linguistics of gradable adjectives. Our main conclusion here was that given these results, the bridge principle in question cannot be maintained and the supposed argument against the conciliatory position is unsound. Thus, given the existing state of the debate, we don't have good reasons to reject the conciliatory view. If what precedes is on the right track, then we may well be in a position to end the decades-old stalemate between Achinstein and the probabilists and move the debate about evidence and confirmation onwards.

\section{REFERENCES}

Achinstein, Peter (1978). Concepts of evidence. Mind 87(345): 22-45.

Achinstein, Peter (1981). On evidence: a reply to Bar-Hillel and Margalit. Mind 40: 108-112.

Achinstein, Peter (1983). The Concept of Evidence. Oxford University Press.

Achinstein, Peter (1992). The evidence against Kronz. Philosophical Studies 67: 169-175.

Achinstein, Peter (1994). Stronger evidence. Philosophy of Science 61 (3):329-350. 
Achinstein, Peter (1996). Swimming in evidence: a reply to Maher. Philosophy of Science 63(2): $175-182$.

Achinstein, Peter (2000). Why philosophical theories of evidence are (and ought to be) ignored by scientists. Philosophy of Science 67(3): 192.

Achinstein, Peter (2001). The Book of Evidence. Oxford University Press.

Achinstein, Peter (2004). A challenge to positive relevance theorists: reply to Roush. Philosophy of Science 71(4): 521-524.

Achinstein, Peter (2013). Evidence and Method: Scientific Strategies of Isaac Newton and James Clerk Maxwell. Oxford University Press.

Bar-Hillel, Maya and Margalit, Avishai (1979). In defense of the classical notion of evidence. Mind 88: 576-583.

Bartsch, R., and Vennemann, T. (1972). Semantic Structures: A Study in the Relation between Semantics and Syntax. Athenäum.

Carnap, Rudolf (1962). Logical Foundations of Probability. University of Chicago Press.

Cresswell, M. J. (1976). The semantics of degree. In Montague Grammar (pp. 261-292).

Glanzberg, Michael (2014). Explanation and partiality in semantic theory. In A. Burgess and B. Sherman (eds.) Metasemantics: New Essays on the Foundations of Meaning. Oxford University Press.

Grice, H.P. (1975). Logic and conversation. In Syntax and semantics, volume 3, Speech acts, eds. P. Cole and J.L. Morgan, 41-58. New York: Academic Press.

Hájek, Alan, and Joyce, James M. (2008). Confirmation. In S. Psillos and M. Curd (eds.) The Routledge Companion to the Philosophy of Science. Routledge.

Heim, I. (1985). Notes on comparatives and related matters. Unpublished ms., University of Texas, Austin.

Howson, Colin, and Urbach, Peter (2006). Scientific Reasoning: The Bayesian Approach. Open Court.

Kelly, Thomas (2014). Evidence. In Edward N. Zalta (ed.) The Stanford Encyclopedia of Philosophy (Fall 2014 Edition). URL = 〈http://plato.stanford.edu/archives/fall2014/entries/evidence/>

Kennedy, Christopher (1999). Projecting the Adjective. Ph.D. dissertation, University of California, Santa Cruz. Published by Garland.

Kennedy, Christopher (2007). Vagueness and grammar: the semantics of relative and absolute gradable adjectives. Linguistics and Philosophy 30(1): 1-45.

Kennedy, Christopher, and McNally, Louise (2005). Scale structure, degree modification, and the semantics of gradable predicates. Language 81(2): 345-381. 
Kronz, Frederick M. (1992). Carnap and Achinstein on evidence. Philosophical Studies 67: 151-167. Maher, Patrick (1996). Subjective and objective confirmation. Philosophy of Science 63(2): 149-174. Maher, Patrick (2005). Confirmation theory. In The Encyclopedia of Philosophy, 2nd ed. Macmillan. Roush, Sherrilyn (2004). Discussion note: positive relevance defended. Philosophy of Science 71(1): $110-116$.

Rotstein, C., and Winter, Y. (2004). Total adjectives vs. partial adjectives: scale structure and higherorder modification. Natural Language Semantics 12: 259-288.

Salmon, Wesley C. (1973). Confirmation. Scientific American 228: 75-83.

Salmon, Wesley C. (1983). Confirmation and relevance. In Peter Achinstein (ed.) The Concept of Evidence. Oxford University Press.

Unger, Peter (1975). Ignorance. Oxford University Press. 